\begin{tabular}{l|l} 
Ebisu & Ebisu \\
Études japonaises & Études japonaises
\end{tabular}

$54 \mid 2017$

L'après-guerre des intellectuels japonais

\title{
À propos des numéros spéciaux « Japon »
}

Cécile Sakai

\section{OpenEdition}

\section{Journals}

Édition électronique

URL : http://journals.openedition.org/ebisu/2140

DOI : $10.4000 /$ ebisu. 2140

ISSN : 2189-1893

\section{Éditeur}

Institut français de recherche sur le Japon à la Maison franco-japonaise (UMIFRE 19 MEAE-CNRS)

\section{Édition imprimée}

Date de publication : 19 décembre 2017

Pagination : 251-255

ISSN : 1340-3656

\section{Référence électronique}

Cécile Sakai, «À propos des numéros spéciaux « Japon » », Ebisu [En ligne], 54 | 2017, mis en ligne le 19 décembre 2017, consulté le 23 septembre 2020. URL : http://journals.openedition.org/ebisu/2140 DOI : https://doi.org/10.4000/ebisu.2140 
Livres à lire 



\section{Comptes rendus}

\section{À propos des numéros spéciaux " Japon »}

Les amateurs éclairés ont pu constater au cours de l'année 2017 la parution successive de plusieurs numéros spéciaux consacrés au Japon, dans des revues généralistes ou disciplinaires réputées et inscrites dans différents champs de la réflexion intellectuelle en France. Le comité éditorial d'Ebisu, revue d'études japonaises créée en 1993 à la Maison franco-japonaise à Tokyo (classée par l'AERES en
2008), ne peut que se réjouir, dans son $54^{\mathrm{e}}$ numéro, d'une telle situation. Depuis quelques années d'ailleurs, les questions japonaises sont bien présentes dans des revues générales: si les circonstances tragiques de la triple catastrophe du Japon du Nord-Est ne sont pas étrangères à certains choix éditoriaux depuis 2011, l'intérêt pour le Japon, en tant qu' "objet thématique " dépasse cet horizon. Tout se passe comme si, après une longue période d'indifférence, les responsables des publications avaient décidé de se pencher sur les problématiques aréales, et spécifiquement celles du Japon.

$\mathrm{Ne}$ nous méprenons pas cependant: derrière cette tendance, 
s'activent des spécialistes convaincus, qui ne ménagent pas leur peine pour faire connaître leurs travaux et ceux de leurs pairs, qui sélectionnent et traduisent en français des études écrites par leurs collègues japonais, qui sans relâche cherchent à mieux insérer l'« objet japonais » parmi les paradigmes généraux de la connaissance scientifique. Mais leurs efforts ne porteraient pas leurs fruits s'il n'y avait pas du côté de la réception un intérêt culturel accru pour le Japon, aujourd'hui espace des esthétiques alternatives, renvoyant à l'idée de "moderne sans être occidental", pour citer le titre de la récente étude historique de Pierre-François Souyri ${ }^{1}$. Lentement mais sûrement, l'on assiste à la construction d'un objet général de la connaissance, qui a pour nom "Japon ", autour de débats sur les modèles et contre-modèles, sur le décentrement comme approche herméneutique, sur la connexion et le dialogue, pour une vision scientifique plurielle et mieux partagée.

Autant d'occasions en tous les cas qui permettent de désenclaver les recherches, de les offrir en lecture à d'autres spécialistes d'autres champs, de se confronter à leurs analyses et à leurs regards critiques. La diffusion bénéficie de plus d'un réel développement grâce aux supports numériques, que les revues soient déjà au format sur des portails tels que Persée ou
Revues.org, ou qu'elles fassent partie de différents bouquets numériques, notamment BibCNRS et Cairn. Autrement dit, l'accès libre et global représente en soi une chance supplémentaire d'ouvrir les frontières de la spécialisation - tout en conservant bien évidemment la haute expertise des données locales, indispensable aux recherches aréales. Il reste enfin la question de la langue de publication : la francophonie doit rester centrale dans une vision multiculturelle et multilinguistique du savoir. Mais cela n'exclut pas les interfaces avec l'anglais, avec au minimum de brefs résumés, voire des sélections d'articles traduits en anglais; et l'on constate aussi par les statistiques d'accès aux revues numériques que le format est réellement incitatif dans tous les continents, y compris des régions non francophones.

Citons quelques numéros récents :

- Revue Critique, avril 2017, tome LXXIII, $n^{\circ} 839$ : «Et le Japon devint moderne " https://www.cairn. info/revue-critique.htm

- Revue Médiévales, printemps 2017: "Roman du Genji et société aristocratique au Japon» https://www.cairn.info/revue-medievales-2017-1.htm

- Revue philosophique, $\mathrm{n}^{\circ} 1$, janvier-mars 2017 : «Philosopher au Japon, 2 " https://www.cairn.info/ revue-philosophique-2017-1.htm 
- Revue Histoire, Économie \& Société, no 2, juin 2017 : «Mibun Penser les statuts sociaux du Japon prémoderne (XvI ${ }^{\mathrm{e}}$-xIX ${ }^{\mathrm{e}}$ siècles)» https:// www.cairn.info/revue-histoire-economie-et-societe-2017-2.htm

À paraître début 2018 :

- Revue Tracés. Revue de Sciences Humaines Hors série 2017 "Traduire et introduire les sciences sociales d'Asie Orientale" (articles sur le Japon, la Corée, la Chine) http:/traces.revues.org

Nous ne chercherons pas ici à commenter chacune de ces parutions, mais elles composent ensemble un kaléidoscope pluridisciplinaire, en histoire, littérature, philosophie, anthropologie, etc. Aux côtés des deux revues francophones spécialisées en études japonaises ${ }^{2}$ que sont Ebisu - Études japonaises à Tokyo et Cipango-Cahiers d'etudes japonaises ${ }^{3}$, fondée à Paris en 1992 par le département d'études japonaises de l'Inalco, elles offrent des possibilités nouvelles de coordination. Signalons aussi le lancement en juillet 2017 d'un tout nouveau blog scientifique de publication, adossé à la Société Française d'Etudes Japonaises (SFEJ), intitulé Japon(s) Carnet de la SFEJ, sur https://sfej.hypotheses.org. Cette formule relativement souple, sous la houlette d'un jeune comité de rédaction, doit faciliter aussi le partage des connaissances et des informations.
N'oublions pas enfin les revues régionales sur l'Asie, qui peuvent aussi porter des dossiers sur le Japon. On rappellera principalement ExtrêmeOrient Extrême-Occident ${ }^{4}$, dont le dernier numéro $(41,2017)$ sur les : "Statuts et identités dans l'Asie prémoderne $\mathrm{XVII}^{\mathrm{e}}$-XIX ${ }^{\mathrm{e}}$ siècles" comprend ainsi plusieurs articles sur le Japon.

Désenclavement et amplification de la réception : le tableau paraîtrait presque idyllique s'il n'y avait pas toute une série de difficultés inhérentes à la production des revues. Tout d'abord le travail considérable de préparation de chaque numéro, devant obéir désormais aux critères des évaluations scientifiques : appels à projet, rapports externes anonymes, long travail d'élaboration entre les auteurs et les comités de rédaction, jusqu’à la version finale. $\mathrm{Au}$ plus rapide, la préparation d'un numéro nécessite 18 mois de travail et de multiples relectures, de la part d'équipes formées d'enseignantschercheurs assumant une multitude d'autres missions fondamentales dans l'enseignement et la recherche, et le plus souvent sans soutien technique.

Le dernier numéro de la revue trimestrielle Perspectives chinoises/ China Perspectives, $\mathrm{n}^{\circ} 2017 / 3$, éditée en français et en anglais par le Centre français d'Études sur la Chine Contemporaine (CEFC, 
UMIFRE 18 CNRS/MEAE), s'ouvre sur un éditorial fort instructif de Séverine Arsène, rédactrice en chef de la revue, qui présente son bilan de quatre années à ce poste, sous l'intitulé : «Perspectives chinoises au cœur des mutations scientifiques et numériques ». Tout en se félicitant de la récente inscription de la revue, classée par l'AERES en 2011, à l'Emerging Sources Citation Index, après l'index Scopus en 2015, l'auteure de l'éditorial alerte sur les biais et dérives induits par un "certain modèle managérial d'abord répandu dans les sciences exactes, privilégiant l'usage d'indicateurs quantitatifs étroits par rapport à une vision plus holistique de l'activité scientifique " (p. 4). Accompagné d'une solide bibliographie critique, cet éditorial met en garde contre les effets pervers d'un système d'évaluation hypernormatif, fondé sur l'unique modèle anglo-saxon des "impact factors" en sciences exactes. Il appelle de ses vœux des formules mieux adaptées et diversifiées, qui fassent la part nécessaire aux approches qualitatives et originales - l'un des cœurs du métier en sciences humaines et sociales. La négociation de ce vaste champ de recherche avec la mutation numérique en cours représente un enjeu fondamental pour l'avenir.

Une autre question se pose aussi, en filigrane, du choix des modèles de publication en SHS : revues ou monographies? Idéalement, les auteurs devraient pouvoir assumer tous les formats, tous azimuts. Le temps dont ils disposent néanmoins n'est pas infini. Si l'on se réfere à l'histoire des SHS au cours du $\mathrm{xx}^{\mathrm{e}}$ siècle, ce sont les monographies qui ont été centrales dans le dispositif de production et transmission des connaissances. L'écriture d'articles plus courts, plus rapides, plus maniables, aurait-elle remplacé, sous la pression du régime d'évaluation, le livre d'auteur? Au nom de quelle valeur scientifique ? Seule sans doute une vision à long terme de la production scientifique en SHS permettra-t-elle de porter un regard possiblement objectif sur les décennies 2000-2020, avec un effet rétroactif dont les leçons, hélas, ne pourront s'appliquer que pour les décennies suivantes.

Il reste que tous les moyens scientifiques de rendre comparable l'objet «Japon» sont aujourd'hui mis en œuvre, avec la collaboration d'un grand nombre d'acteurs engagés. On citera un extrait de l'éditorial du numéro 839 de la revue Critique, susmentionnée: "Le Japon issu de la Restauration de Meiji a longtemps été décrit comme un mélange de "modernité" (occidentale) et de "tradition" (autochtone), mais ce paradigme rudimentaire interdisait de penser toute dynamique proprement 
historique. On comprend mieux aujourd'hui que le Japon a inventé sa version spécifique de la grammaire du moderne, et que c'est au prisme des autres nations qu'il a refaçonné ses héritages" (p. 275). Ce constat, que l'on peut largement partager, autorise de nouvelles approches, confrontations et conversations, toujours plus nombreuses et fructueuses. La revue Ebisu espère contribuer à cette ouverture, en associant les études des spécialistes francophones à celles des grands auteurs japonais, insuffisamment connus et reconnus en France, avec pour mission de les présenter dans leur contexte et - nécessairement - de proposer en traduction leurs textes fondamentaux.

Cécile SAKAI Université Paris Diderot, Umifre 19-MFJ

1. Pierre-François Souyri, Moderne sans être occidental - Aux origines du Japon d'aujourd'hui, Paris, NRF, Gallimard, 2016. Voir le compte rendu dans ce numéro.

2. On rappellera le rôle important joué par la revue Daruma, dont 13 numéros sont parus entre 1997 et 2003, sous la houlette des responsables du département de japonais de l'université de Toulouse. Durant 7 années, trois revues francophones spécialisées se sont donc côtoyées, chacune avec ses caractéristiques.

3. 21 numéros et 3 hors série parus. Voir https://cipango.revues.org/, qui donne également accès à une sélection en anglais : Cipango - Japanese Studies: English Edition

4. https://extremeorient.revues.org/

Pierre-François Souyri, Moderne sans être occidental - Aux origines du Japon d'aujourd'hui, Paris, coll. "Bibliothèque des histoires ", Gallimard, 2016, 496 p.

Voici un ouvrage dont une des qualités premières est d'offrir un panorama et une synthèse remarquables sur les idéologies modernes du Japon.

Moderne sans être occidental: adoptant ici encore la démarche qui le distingue depuis ses travaux sur le Moyen Âge japonais, son auteur, Pierre-François Souyri - professeur à l'université de Genève -, s'emploie à restituer dans notre représentation du Japon le dynamisme historique dont nous avons trop peu conscience. Car si la richesse culturelle de ce pays a toujours fasciné, nous savons peu de choses de sa modernité, sinon, et c'est même un poncif, qu'il a su garder ses traditions malgré sa modernisation fulgurante. Celle-ci n'est vue le plus souvent que comme le résultat de l'assimilation rapide de facteurs exogènes.

Pierre-François Souyri nous invite à nous départir de cet eurocentrisme. Il note que, contrairement 